\title{
Brain Magnetic Resonance Imaging in Patients with Favorable Out- comes after Out-of-Hospital Cardiac Arrest: Many Have Encephalopa- thy Even with a Good Cerebral Performance Category Score
}

\author{
Woo Sung Choi, M.D., Jin Joo Kim, M.D., and Hyuk Jun Yang, M.D. \\ Department of Emergency Medicine, Gachon University Gill Medical Center, Incheon, Korea
}

Background: The aim of this study was to retrospectively evaluate and analyze the brain magnetic resonance imaging (B-MRI) findings of patients with a favorable neurological outcome following cerebral performance category (CPC) after out-of-hospital cardiac arrest (OHCA) at single university hospital emergency center.

Methods: Patients with return of spontaneous circulation (> $24 \mathrm{~h}$ ) after OHCA who were older than 16 years of age and who had been admitted to the emergency intensive care unit (EICU) for over a 57-month period between July 2007 and March 2012 and survived with a favorable neurological outcome were enrolled. B-MRI was taken after recovery of their mental status.

Results: Fifty-two patients among the 305 admitted patients had a good CPC, and 33 patients' B-MRI were analyzed (CPC 1: 26 patients, CPC 2: 7 patients). Among these, 18 (54.5\%) patients had a normal finding on B-MRI. On the other hand, ischemia/infarction/microangiopathy compatible with hypoxic-ischemic encephalopathy (HIE) were found on various brain areas including subcortical white matter (7/13), cerebral cortex, central semiovlae, basal ganglia, putamen, periventricular white matter, and cerebellum.

Conclusions: Survivors with a favorable neurological outcome from OHCA showed HIE on B-MRI, especially all of the patients with a CPC 2. More detail neurologic category including brain imaging would be needed to categorize patients with favorable outcome after OHCA.

Key Words: magnetic resonance imaging; outcome; out-of-hospital cardiac arrest; therapeutic hypothermia.

\section{Introduction}

Out-of-hospital cardiac arrest (OHCA) is fatal thus is a major global health concern. There were approximately 166-200 OHCA cases in the U.S. in 2008,[1] and the standardized incidence rates in South Korea in 2006 and 2007 were 20.9 and 22.2 per 100,000, respectively.[2] The survival discharge rate was extremely low (3.5\%) for the resuscitation-attempted group, and only $1.1 \%$ of all the patients had a favorable neurological outcome (cerebral performance category [CPC] 1-2).[2] Cardiac arrest results in global cerebral ischemia as well as hypoxic-ischemic encephalopathy (HIE) even for patients with a good CPC. The neuropsychological sequelae of HIE are memory disturbances, amnesia, insomnia, change of personality and behavior,

Received on May 29, 2015 Revised on August 11, 2015

Accepted on August 19, 2015

Correspondence to: Jin Joo Kim, Department of Emergency Medicine, Gachon University Gil Medical Center, 21 Namdong-daero 774beon-gil, Namdong-gu, Incheon 21565, Korea

Tel: +82- 32-460-2080, Fax: +82- 32-460-3019

E-mail: empear@giilhospital.com

*No potential conflict of interest relevant to this article was reported. visual deficit, language skills, and so on.[3] But there were few studies about HIE of patients with a favorable outcome. Roine et al[4] reported that $60 \%$ of patients that had survived from arrest had severe cognitive deficits at 3 months and $48 \%$ at 12 months after insult.

The aim of this study was to retrospectively evaluate and analyze the brain magnetic resonance imaging (B-MRI) find-

cc This is an Open Access article distributed under the terms of the Creative Commons Attribution Non-Commercial License (http://creativecommons.org/ licenses/by-nc/3.0/) which permits unrestricted non-commercial use, distribution, and reproduction in any medium, provided the original work is properly cited. 
ings of patients with a favorable neurological outcome following CPC after out-of-hospital cardiac arrest at one emergency center.

\section{Materials and Methods}

\section{1) Study population}

The study institution was a 1,600-bed university hospital with an annual emergency intensive care unit (EICU) census of 1,000 . This was a retrospective study of patients with return of spontaneous circulation $(>24 \mathrm{~h}$ ) after OHCA who were older than 16 years of age and who had been admitted to the EICU for over a 57-month period between July 2007 and March 2012. After admission, patients received post cardiac arrest care including therapeutic hypothermia (TH) following a standardized protocol. Patients with a favorable neurological outcome (CPC 1-2) underwent B-MRI after recovery of their mental status and then enrolled in our study. CPC were measured at 6 month after discharge. Patients who did not receive TH and did not undergo B-MRI, patients who were pregnant, traumatic cardiac arrest were excluded from the study. Informed consent was received for the study following the protocol used in the authors' department. In the authors' emergency center, the standardized post cardiac arrest care protocols have been used since 2007 and are updated annually.

\section{2) B-MRI}

B-MRI was checked after recovery of consciousness and stabilization of their general condition. B-MRIs available at our institution during the study period were 1.5 Tesla (T) (Magnetom Vision, Siemens, Erlangen, Germany) and 3T scanners (Magnetom Verio, Siemens, Erlangen, Germany). $\mathrm{B}-\mathrm{MRI}$ results were analyzed by neurological radiologists.

\section{Results}

During the study period, a total of 402,607 patients visited our emergency department; 1,145 patients received cardiopulmonary resuscitation, 305 patients were admitted to the EICU and received post cardiac arrest care, 226 patients survived, 52 patients had a good CPC, and 33 had a B-MRI

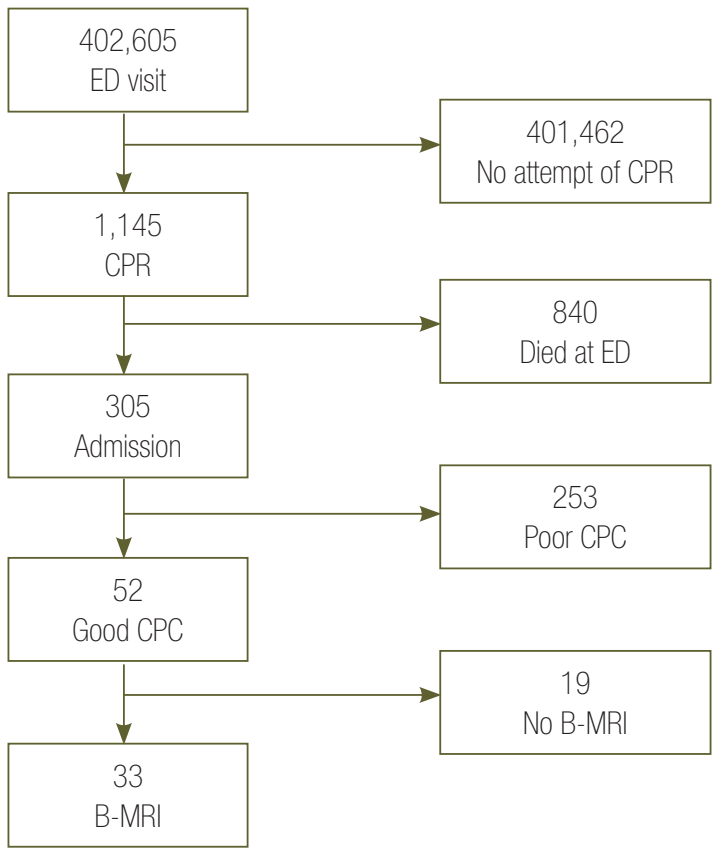

Fig. 1. This diagram shows the enrolled patients during July 2007 to March 2012. ED: emergency department; CPR: cardiopulmonary resuscitation; CPC: cerebral performance category; B-MRI: brain magnetic resonance imaging (good CPC: CPC1-2).

Table 1. Basal characteristics of the patients $(N=305)$

\begin{tabular}{lc}
\hline Gender (male) & $210(69.0)$ \\
Age (year) & $53.33( \pm 15.242)$ \\
Smoking (PYR) & $8.84( \pm 16.040)$ \\
\hline Initial rhythm, non-shockable & $217(71.1)$ \\
Shockable & $88(28.9)$ \\
Cause of arrest, cardiac & $193(63.4)$ \\
Non-cardiac disease & $80(26.1)$ \\
Trauma & $32(10.6)$ \\
AR_BLS (min) & $7.33( \pm 5.882)$ \\
AR_ACLS (min) & $18.47( \pm 9.349)$ \\
AR-RO (min) & $35.46( \pm 18.700)$ \\
APACHE II & $24.31( \pm 5.884)$ \\
Lactate (mmol/L) & $9.075( \pm 3.777)$ \\
CPC, good & $52(17.05)$ \\
\hline
\end{tabular}

Data are expressed as mean \pm standard deviation or $n(\%)$.

PYR: pack year; AR_BLS: time from arrest to start the basic life support; AR_ACLS: time from arrest to start the advanced cardiac life support; AR-RO: time from arrest to return of spontaneous circulation; APACHE: acute physiology and chronic health evaluation; CPC: cerebral performance category (good CPC: 1-2, poor CPC: 3-5).

(CPC 1: 26 patients, CPC 2: 7 patients) (Fig. 1). Two patients were excluded due to not having received $\mathrm{TH}$, and 17 
Table 2. Variables by neurologic outcome $(N=305)$

\begin{tabular}{|c|c|c|c|}
\hline & \multicolumn{3}{|c|}{ Neurologic outcome } \\
\hline & Good $(n=52)$ & Poor $(n=253)$ & $\mathrm{p}$-value \\
\hline Male & $39(18.6)$ & $171(81.4)$ & 0.350 \\
\hline Female & $13(13.6)$ & $82(86.4)$ & \\
\hline Age (year) & $50.23( \pm 11.93)$ & $54.50( \pm 16.21)$ & 0.090 \\
\hline Smoking (PYR) & $11.97( \pm 20.25)$ & $7.63( \pm 14.01)$ & 0.224 \\
\hline Initial rhythm, non-shockable & $21(14.9)$ & $117(85.1)$ & $0.000^{*}$ \\
\hline Shockable & $31(58.5)$ & $136(41.5)$ & \\
\hline Cause of arrest, cardiac & $42(37.8)$ & $153(62.2)$ & $0.001^{*}$ \\
\hline Non-cardiac & $10(9.6)$ & $100(90.4)$ & \\
\hline AR_BLS (min) & $5.92( \pm 0.626)$ & $7.88( \pm 0.629)$ & $0.042^{*}$ \\
\hline AR_ACLS (min) & $14.76( \pm 1.292)$ & $19.71( \pm 0.950)$ & $0.003^{*}$ \\
\hline AR-RO (min) & $28.51( \pm 2.812)$ & $38.08( \pm 1.887)$ & $0.006^{*}$ \\
\hline APACHE ॥ & $21.61( \pm 5.22)$ & $25.28( \pm 5.83)$ & $0.001^{*}$ \\
\hline Lactate (mmol/L) & $8.69( \pm 4.12)$ & $9.20( \pm 3.67)$ & 0.561 \\
\hline
\end{tabular}

Data are expressed as mean \pm standard deviation or $n(\%)$.

PYR: pack year, AR_BLS: time from arrest to start the basic life support, AR_ACLS: time from arrest to start the advanced cardiac life support, AR-RO: time from arrest to return of spontaneous circulation, APACHE: acute physiology and chronic health evaluation. ${ }^{*} p<0.05$.

Table 3. Brain MRI findings of the patients with $\operatorname{good} \mathrm{CPC}(\mathrm{N}=33)$

\begin{tabular}{lc}
\multicolumn{1}{c}{ MRI findings } & $\mathrm{n}(\%)$ \\
\hline $\begin{array}{l}\text { Time from admission to scan B-MRI } \\
\text { Days }\end{array}$ & $11.67( \pm 9.00)$ \\
Normal & $18(54.5 \%)$ \\
Abnormal & $13(39.4 \%)$ \\
\hline HIE & \\
Cortex & \\
\hline Temporal lobe & 2 \\
\hline Occipital lobe & 2 \\
\hline Parietal lobe & 2 \\
\hline Frontal lobe & 1 \\
\hline Subcortical WM & 7 \\
Central semiovale & 2 \\
\hline BG, putamen & 3 \\
\hline Cerebellum & 1 \\
\hline PVWM & 2 \\
\hline Underlying abnormalities & $1(3.0 \%)$ \\
\hline Diffuse brain atrophy & $1(3.0 \%)$ \\
\hline Microangiopathy & \\
\hline Dara axpressed as mean & \\
\hline
\end{tabular}

Data are expressed as mean \pm standard deviation or $n(\%)$.

MRI: magnetic resonance imaging; CPC: cerebral performance category, BMRI: brain magnetic resonance imaging; HIE: hypoxic-ischemic encephalopathy; WM: white matter; BG: basal ganglia; PVWM: periventricular white matter (good CPC: CPC 1-2). patients were excluded due to not having taken B-MRI after recovery of mental status. Therefore, a total of 33 B-MRI findings were analyzed in the present study. Table 1 shows the basal characteristics of the patients and Table 2 shows the variables statistically significant related to good CPC.

The average time from arrest to undergoing B-MRI was $11.63( \pm 9.00)$ days $(\leq 5$ days: 5 cases, $>6$ days: 28 cases, acute or subacute period). Among these, 18 (54.5\%) patients had a normal finding on B-MRI and all of those patients had a CPC 1. In the patients with abnormal B-MRI findings, ischemia/infarction/microangiopathy compatible with HIE were found in various brain areas. The most common injured area was subcortical white matter (7/13) in our study. Other injured areas were the cerebral cortex, centrum semiovale, basal ganglia, putamen, periventricular white matter, and cerebellum (Table 3). Underlying abnormalities were diffuse brain atrophy and microangiopathy. The typical findings of HIE on B-MRI were well seen on T2 weighted imaging and fluid attenuated inversion recovery (FLAIR) with increased signal intensity (Fig. 2). One patient with a CPC 2 showed HIE on B-MRI with a high signal change in T2 weighted imaging and FLAIR along the cortex and still had remaining HIE on follow-up imaging at six months after arrest (Fig. 3). He suffered from visual disturbances but did 

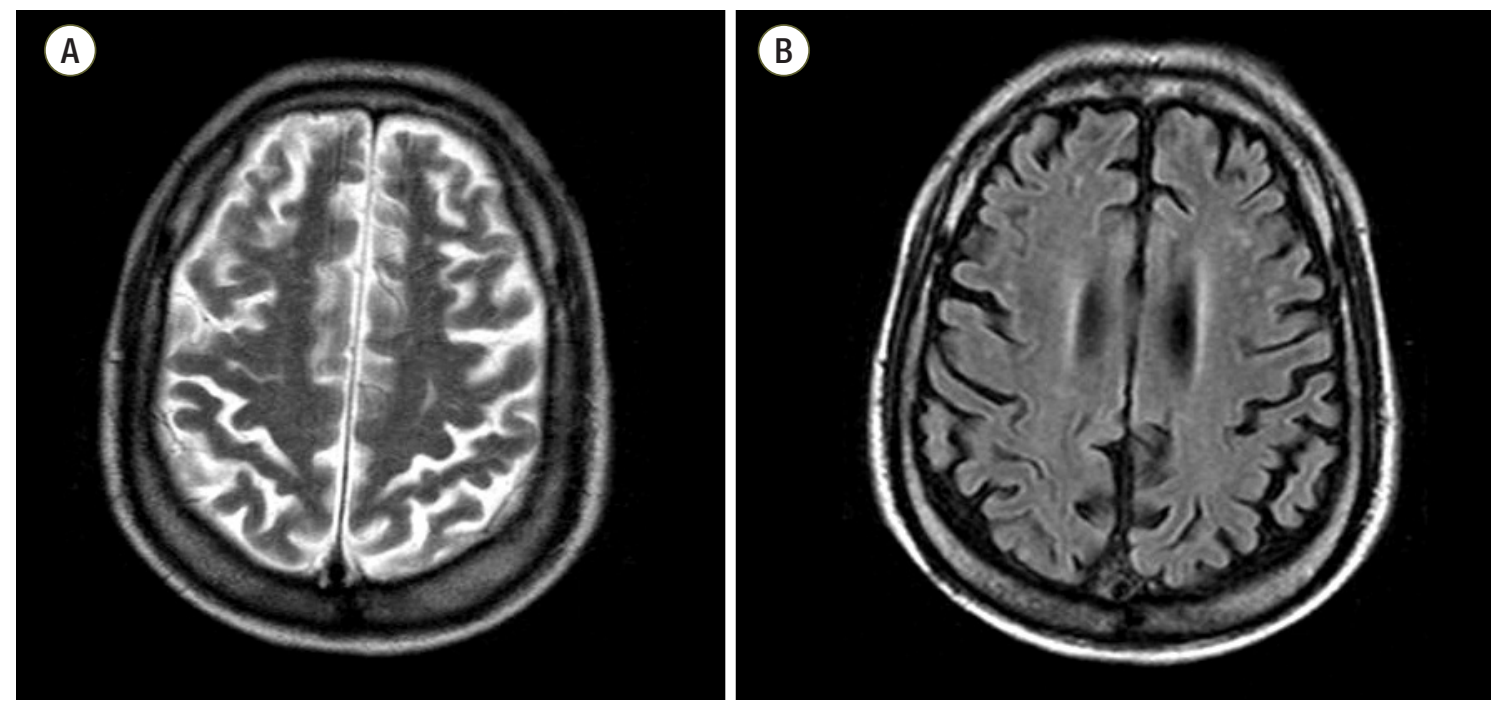

Fig. 2. This brain magnetic resonance imaging shows typical hypoxic-ischemic encephalopathy in subcortical white matter with high signal intensity on T2 weighted imaging (A)/fluid attenuated inversion recovery (B).

usual his life by himself.

\section{Discussion}

Many efforts have been made to overcome the complications of cardiac arrest. The only method proven to improve the neurological outcome after cardiac arrest is TH.[5,6] For this reason, TH is recommended to patients with spontaneous return of circulation after cardiac arrest in 2010 American Heart Association Guidelines for Cardiopulmonary Resuscitation and Emergency Cardiovascular Care.[7] As mentioned earlier, the survival discharge rate was extremely low (3.5\%) for the resuscitation-attempted group, and only $1.1 \%$ of patients had a favorable neurological outcome.[2] Among the patients with a good CPC, HIE could be seen on B-MRI and could have been suffered from various neurological disturbances.

Cortical lesion pattern represents bilateral and symmetrical gyriform involvement through increased signals on T2 weighted imaging and FLAIR. The underlying mechanism of this cortical lesion is suggested to be cortical laminar necrosis. [8] Microvacuolation, which is suggested as the initial phase of cortical laminar necrosis, may reflect the high sensitivity of diffuse weighted imaging through diffusion restriction.[9] Usually physicians check diffusion weighted imaging of brain immediately after cardiac arrest because it takes shorter time to check and could check more briefly in emergency department. Arbelaez et al[10] reported that during the acute period ( $<24$ hours), high-strength diffusionweighted images showed the abnormal basal ganglia, cerebellum, and cortex to a better extent than did conventional MR images. Els et al[11] reported that pathologic diffusion weighted brain imaging during early phase after cerebral hypoxia might be superior to conventional MRI as a predictor of a worse clinical outcome. Diffusion weighted imaging would be better during early stage after hypoxia to predict worse outcome. But in this study, we analyzed conventional MRI during early to subacute state after hypoxia of patients with good outcome.

In the acute period, the cerebral cortex is usually affected in isolation. Involvement of the thalamus, basal ganglia and cerebellum in addition to the cerebral cortex is more frequently seen in scans obtained later cardiac arrest.[12] The hippocampus, medial temporal lobes and basal ganglia are more vulnerable in the condition of pulmonary hypoxic arrest.[13] Topcuoqlu et al[8] reported neurological prognosis based on B-MRI findings, B-MRI of patients in the poor neurological outcome group showed an extensive cortical lesion pattern and those in the good neurological outcome group revealed focal not extensively. In our study, different injured areas were observed on B-MRI including the cortex, 

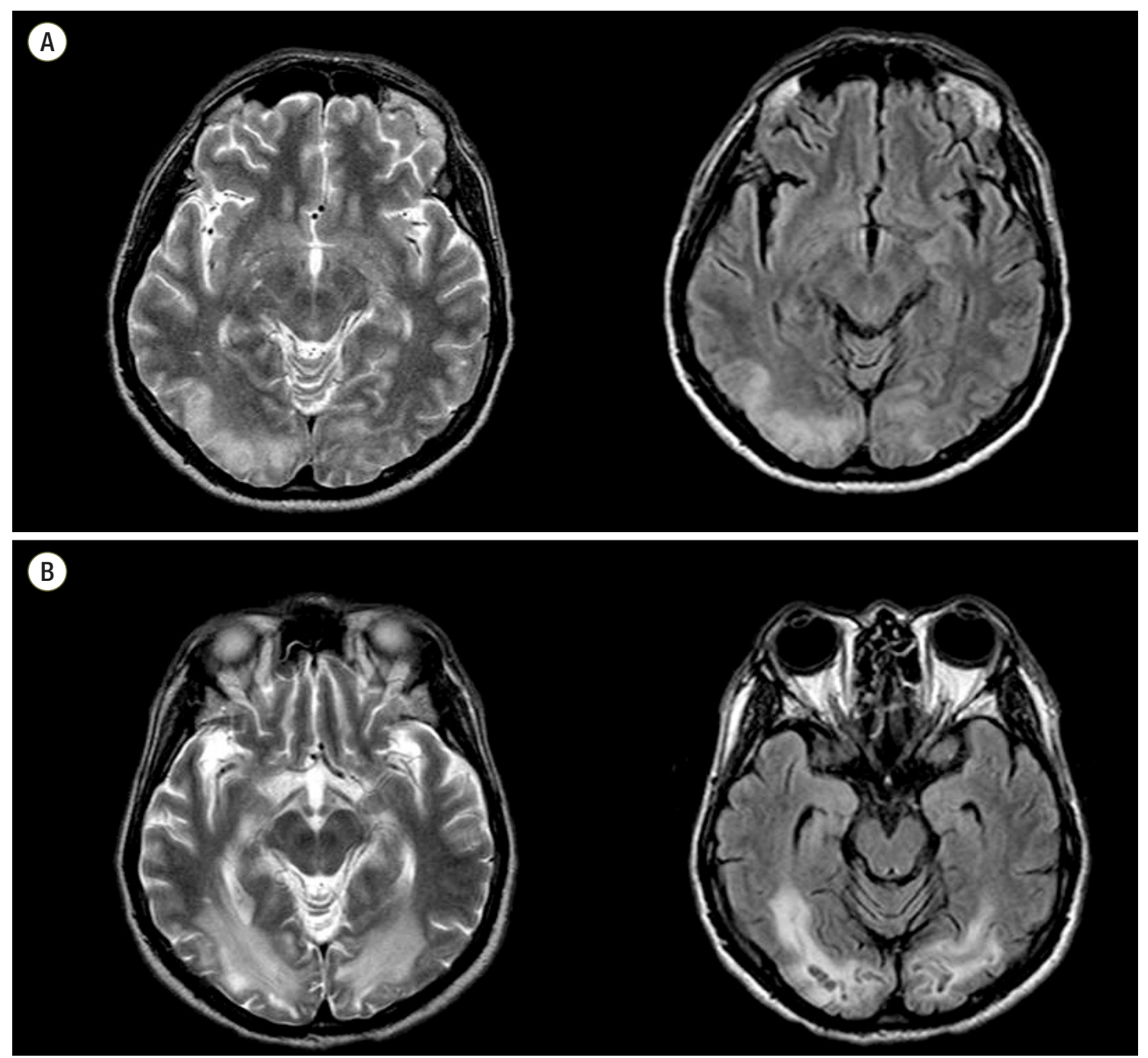

Fig. 3. This brain magnetic resonance imaging shows high signal change in T2 weighted imaging/fluid attenuated inversion recovery along the cortex (A) and follow-up imaging six-month after insult in the patient with cerebral performance category 2 score (B).

subcortical white matter, basal ganglia, cerebellum, and so on. The average time to B-MRI was 11.6 days more after 6 days as subacute period. It is important to note that no typical or fixed pattern of B-MRI abnormality was present in patients with HIE. However, cortical lesion, and other various patterns of B-MRI abnormalities after cardiac arrest have been described especially in diffusion restriction. [13-17] Wu et al[16] reported a retrospective study of 80 patients and showed that whole-brain apparent diffusion coefficients were lower in poor outcome patients than in good outcome patients. Prognostication after cardiac arrest and hypothermia should be performed continuously and cautiously through not only B-MRI but also through clinical neurological examination, serum biomarkers, neurophysiological studies including electroencephalography, somatosensory evoked potential, and so on, even after recovery of mental status.[18,19]

Cognitive impairments in survivors of cardiac arrest have been reported. One prospective study reported cognitive function of 57 patients who survived from OHCA. Among these patients, 11 to $28 \%$ of survivors were cognitively impaired, while $58 \%$ scored unimpaired for all tests. Daily life activities were limited in 19\% of the patients.[20] Grubb et al[21] reported chronic memory impairment especially verbal and spatial memory in 35 survivors of OHCA than controls. In our study, patients with a good CPC also had 
cognitive dysfunction including memory disturbances and visual deficits, even if they showed normal B-MRI findings. All patients with a CPC2 had HIE on B-MRI and most of them had cognitive dysfunction. In general, Glasgow outcome score or CPC are used to check patient's neurologic outcome in post cardiac arrest syndrome. We need more detail neurologic category applied brain imaging to categorize patients with favorable outcome after OHCA and physicians do rehabilitation more actively to these patients. This would be effective to care and upgrade their life ability by rehabilitation program following category. Correlation between cognitive dysfunction and B-MRI findings was not investigated in our study. Further research including neuroimaging, neurophysiologic and neuropsychological studies of survivors after cardiac arrest will be needed. Our study has some limitations. First, since this was a retrospective one center study, a number of unknown biases could have been introduced. Second, the time from arrest to B-MRI varied among the study participants. In our institution usually B-MRI is taken after stabilization after acute stage of post-cardiac arrest care including extubation because it takes time to check full MRI. But ischemic lesion could vary following checked time and if there were very small lesion, it couldn't be seen in imaging.

Survivors with a favorable neurological outcome from OHCA could show HIE on B-MRI, especially all of the patients with a CPC 2. More detail neurologic category applied brain imaging would be needed to categorize patients with favorable outcome after OHCA and physicians do rehabilitation more actively to these patients.

\section{ORCID}

$\begin{array}{ll}\text { Woo Sung Choi } & \text { http://orcid.org/0000-0002-0011-0985 } \\ \text { Jin Joo Kim } & \text { http://orcid.org/0000-0002-5678-2019 } \\ \text { Hyuk Jun Yang } & \text { http://orcid.org/0000-0001-8324-9749 }\end{array}$

\section{References}

1) Rosamon W, Flegal K, Furie K, Go A, Greenlund K, Haase N, et al: Heart disease and stroke statistics--2008 updates: a report from the American Heart Association
Statistics Committee and Stroke Statistics Subcommittee. Circulation 2008; 117: e25-146.

2) Ahn KO, Shin SD, Suh GJ, Cha WC, Song KJ, Kim SJ, et al: Epidemiology and outcomes from non-traumatic out-of-hospital cardiac arrest in Korea: a nationwide observational study. Resuscitation 2010; 81: 974-81.

3) Caine D, Watson JD: Neuropsychological and neuropathological sequelae of cerebral anoxia: a critical review. J Int Neuropsychol Soc 2000; 6: 86-99.

4) Roine RO, Kajaste S, Kaste M: Neuropsychological sequlae of cardiac arrest. JAMA 1993; 269: 237-42.

5) Hypothermia after Cardiac Arrest Study Group: Mild therapeutic hypothermia to improve the neurologic outcome after cardiac arrest. N Engl J Med 2002; 346: 549-56.

6) Bernard SA, Gray TW, Buist MD, Jones BM, Silvester W, Gutteridge G, et al: Treatment of comatose survivors of out-of-hospital cardiac arrest with induced hypothermia. N Engl J Med 2002; 346: 557-63.

7) Peberdy MA, Callaway CW, Neumar RW, Geocardin RG, Zimmerman JL, Donnino M, et al: Part 9: postcardiac arrest care: 2010 American Heart Association Guidelines for Cardiopulmonary Resuscitation and Emergency Cardiovascular Care. Circulation 2010; 122(18 Suppl 3): S768-86.

8) Topcuoqlu MA, Oquz KK, Buyukserbetci G, Bulut E: Prognostic value of magnetic resonance imaging in post-resuscitation encephlaopathy. Intern Med 2009; 48: 1635-45.

9) Kawahara H, Takeda Y, Tanaka A, Nagano O, Katayama $\mathrm{H}$, Hirakawa $\mathrm{M}$, et al: Does diffusion-weighted magnetic resonance imaging enable detection of early ischemic change following transient cerebral ischemia? J Neurol Sci 2000; 181: 73-81.

10) Arbelaez A, Castillo M, Mukherji SK: Diffusionweignted MR imaging of global cerebral anoxia. AJNR Am J Neuroradiol 1999; 20: 999-1007.

11) Els T, Kassubek J, Kubalek R, Klisch J: Diffusionweighted MRI during early global cerebral hyoxia: a predictor for clinical outcome? Acta Neurol Scand 2004; 110: 361-7.

12) Weiss N, Galanaud D, Carpentier A, Naccache L, Puybasset L: Clinical review: prognostic value of magnetic resonance imaging in acute brain injury and coma. Crit 
Care 2007; 11: 230.

13) Singhal AB, Topcuoqlu MA, Koroshetz WJ: Diffusion MRI in three types of anoxic encephalopathy. J Neurol Sci 2002; 196: 37-40.

14) Greer D, Scripko P, Bartscher J, Sims J, Camargo E, Singhal A, et al: Serial MRI changes in comatose cardiac arrest patients. Neurocrit Care 2011; 14: 61-7.

15) Wijdicks EF, Campeau NG, Miller GM: MR imaging in comatose survivors of cardiac resuscitation. AJNR Am J Neuroradiol 2001; 22: 1561-5.

16) Wu O, Sorensen AG, Benner T, Singhal AB, Furie KL, Greer DM, et al: Comatose patients with cardiac arrest: predicting clinical outcome with diffusion-weighted MR imaging. Radiology 2009; 252: 173-81.

17) Konaka K, Miyashita K, Naritomi H: Changes in diffusion-weighted magnetic resonance imaging findings in the acute and subacute phases of anoxic enecephalopathy. J Stroke Cerebrovasc Dis 2007; 16: 82-3.

18) Friberg H, Rundgren $M$, Westhall E, Nielsen N, Cronberg T: Continuous evaluation of neurological prognosis after cardiac arrest. Acta Anaesthesiol Scand 2013;57:6-15.

19) Samaniego EA, Persoon S, Wijman CA: Prognosis after cardiac arrest and hypothermia: a new paradigm. Curr Neurol Neurosci Rep 2011; 11: 111-9.

20) van Alem AP, de Vos R, Schmand B, Koster RW: Cognitive impairment in survivors of out-of-hospital cardiac arrest. Am J Heart 2004; 148: 416-21.

21) Grubb NR, O'Carroll R, Cobbe SM, Sirel J, Fox KA: Chronic memory impairment after cardiac arrest outside hospital. BMJ 1996; 313: 143-6. 